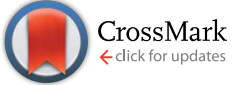

Cite this: Chem. Sci., 2017, 8, 340
Received 5th July 2016

Accepted 11th August 2016

DOI: $10.1039 / \mathrm{c} 6 s c 02956 f$

www.rsc.org/chemicalscience

\section{Janus second-order nonlinear optical dendrimers: their controllable molecular topology and corresponding largely enhanced performance $\uparrow$}

\author{
Runli Tang, $\ddagger^{\mathrm{a}}$ Shengmin Zhou, $\$^{\mathrm{a}}$ Ziyao Cheng, ${ }^{\mathrm{a}}$ Gui Yu, ${ }^{\mathrm{b}}$ Qian Peng, ${ }^{\mathrm{b}}$ Huiyi Zeng, ${ }^{\mathrm{c}}$ \\ Guocong Guo, ${ }^{c}$ Qianqian $\mathrm{Li}^{\mathrm{a}}$ and Zhen $\mathrm{Li}^{* a}$
}

\begin{abstract}
A new type of Janus dendrimers, consisting of two different side dendrons with the dipole orientation of the second-order nonlinear optical (NLO) chromophore moieties partially in a non-centrosymmetric direction, was intelligently designed and synthesized in order to enhance the macroscopic NLO performance and break through the limitation of NLO efficiency in the current molecular topological structure of azo chromophore-based polymers. This kind of Janus dendritic structure was constructed by the combination of convergent and divergent methods, with the utilization of a powerful "click chemistry reaction". The obtained three dendrimers, D-13N, D-17N and D-21N, show very high NLO performance, especially the dramatically enhanced NLO coefficient of $299 \mathrm{pm} \mathrm{V}^{-1}$ for D-13N, which is the highest value ever reported for polymers containing a simple azo chromophore. The new dendrimers provide a clear structure-properties relationship between high NLO efficiency and the controllable molecular topology with the non-centrosymmetrical alignment of dipole orientation, thus opening up a new avenue for the further development of NLO dendrimers with high performance and more importantly providing some clues for the rational design of functional dendrimers with controllable molecular topology.
\end{abstract}

\section{Introduction}

The architectural control of polymeric molecules is an important aspect in the field of polymer science, which to a large extent determines their diverse properties. ${ }^{1}$ Thus, architectural control is regarded as the switch connecting designed structures and their corresponding specific properties, which has triggered much inspiration of scientists. ${ }^{2}$ Among them, dendrimers with regular branching and symmetry are one of the most interesting structures; because they have a unique topological structure, dendrimers are equipped with many superb properties, and have brought about numerous advances in a variety of fields. ${ }^{3}$ In addition to their regular and symmetry features, dendrimers

\footnotetext{
a'Department of Chemistry, Wuhan University, Wuhan 430072, China. E-mail: lizhen@ whu.edu.cn; Fax: +862768756757

${ }^{b}$ Institute of Chemistry, The Chinese Academy of Sciences, Beijing 100080, China

${ }^{c}$ Fujian Institute of Research on the Structure of Matter, The Chinese Academy of Sciences, Fuzhou 350002, China

$\dagger$ Electronic supplementary information (ESI) available: The structures of dendrimers in the previous study and the related parameters, the synthesis of compounds, NLO measurements including NLO coefficient theories, the preparation of thin films, measurement condition and error analysis, molecular mechanics simulation including calculation method and results, NMR spectra, TOF mass spectra, UV-vis absorption spectra, TGA and DSC thermograms, and UV-vis absorption of thin films before and after poling. See DOI: 10.1039/c6sc02956f

\$ These authors contributed equally to this manuscript.
}

often possesses more than one valuable property derived from different functional groups located at different sites of internal dendrons, layer or terminal groups. ${ }^{4}$ Some specific dendrimers, classified as bow-tie or Janus, ${ }^{5}$ three-face, ${ }^{6}$ core-shell, ${ }^{7}$ onion peel, ${ }^{8}$ etc., are attractive not only for their amazing functions or properties, but also for the art of their syntheses. A dendrimer with two dendritic wedges coupled together with a formed core, is called a Janus or bow-tie dendrimer (Fig. 1a). "Janus" was the name of the mythological two-faced god who looked to the future and past in ancient Roman religion., ${ }^{5,9}$

Generally, Janus dendrimers focus their asymmetry on the surface functionality, as the two dendrons connect two different types of peripheral functional groups. The dual-functionalized surfaces endow the dendrimers with different properties, which has allowed them be widely used in self-assembly ${ }^{\mathbf{1 0}}$ and biological therapeutics. ${ }^{\mathbf{1 1}}$ However, their applications in optoelectronic materials are very scarce until now, ${ }^{\mathbf{1 2}}$ partially because the photo- and electronic properties mainly depend on the electronic structure of the repeat unit instead of the peripheral groups. Nevertheless, we guess that a Janus dendrimer would possess huge potential advantages in this area, for at least, the asymmetric features of "Janus" exactly meets the requirement of a non-centrosymmetric alignment of chromophore moieties in second order nonlinear optical (NLO) polymers, which has never been reported previously.

Second order NLO polymers have found numerous applications in photonic devices, such as high speed electro-optic (EO) 
a) Janus dendrimer

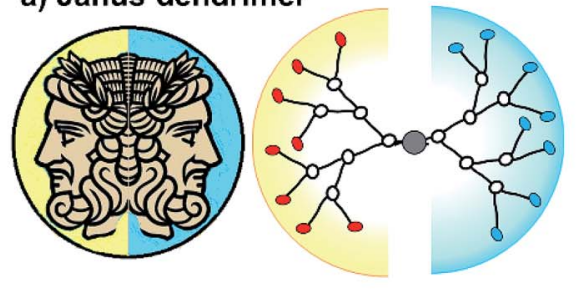

b) Poling process

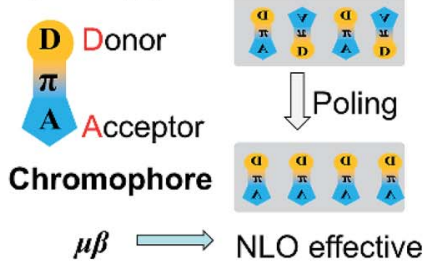

c) NLO dendrimer

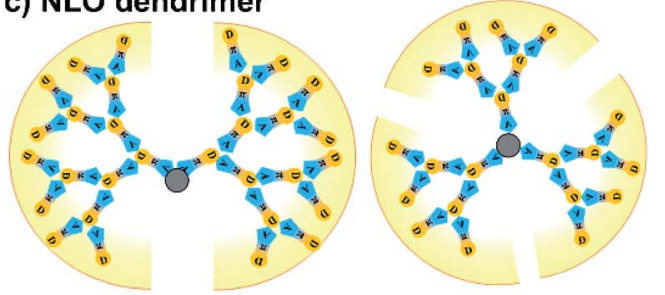

\section{d) Janus NLO dendrimer}

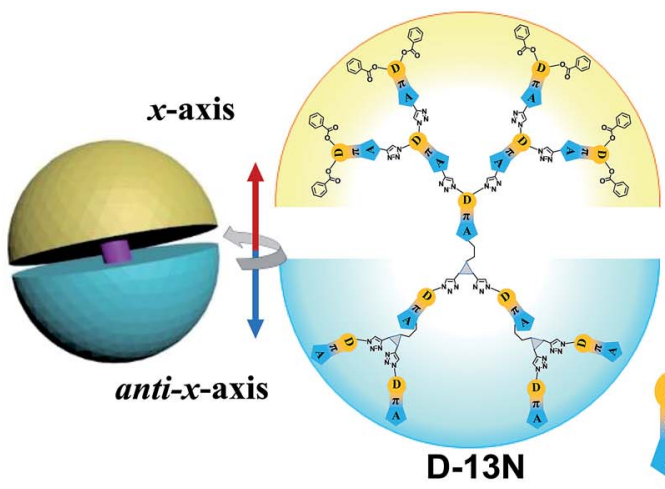

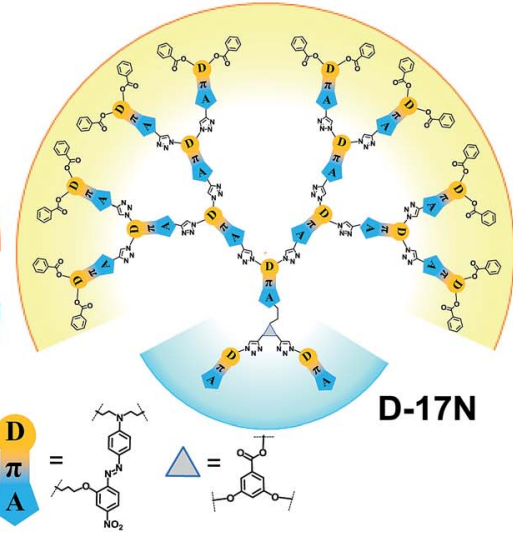

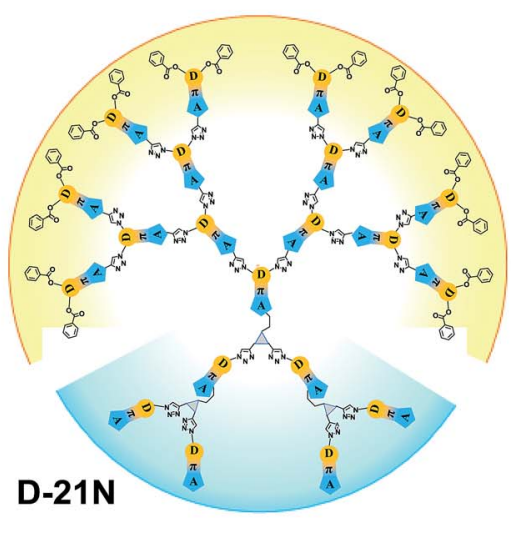

Fig. 1 (a) Janus dendrimer, (b) NLO chromophore and poling process, (c) NLO dendrimers and (d) Janus NLO dendrimers.

modulators, ${ }^{13}$ waveguides, ${ }^{14}$ optical switches,${ }^{15}$ etc. ${ }^{16}$ Since organic/polymer NLO materials initially came to the attention of researchers for electro-optic devices, they have attracted intense interest with much impressive progress being made, due to their advantages over inorganic crystals. ${ }^{16,17}$ However, the bottleneck encountered in the following research, that is, how to fully optimize the NLO performance, has cooled research interest since the end of the last century.

Generally, a second order NLO polymer is composed of polymer chains and chromophore moieties; the latter of which possesses an asymmetric pull-push electronic structure (Fig. 1b), which contributes to its dipole moment $(\mu)$ and hyperpolarizability $(\beta)$. Since the macroscopic second order NLO effect of a polymer is the sum of the $\mu \beta$ values of chromophore moieties in a non-centrosymmetric alignment (for brief theory see the ESI $\dagger),{ }^{18}$ a poling process under an electric field is usually implemented to make the chromophore moieties in polymers realign from the natural centrosymmetric state into a non-centrosymmetric state as "poled polymers"19 (Fig. 1b). In order to transfer the microscopic $\mu \beta$ of a chromophore into a large macroscopic NLO coefficient to the largest extent, numerous polymers were constructed to figure out some guidelines. ${ }^{17-20}$ Among them, dendrimers demonstrated their absolute superiority in having a much higher NLO coefficient in comparison with other polymers. ${ }^{21}$ Many NLO dendrimers (i) containing different chromophores, (ii) in varied forms like chromophore (as a repeat unit) construction or multiple dendron modified chromophore, (iii) in unique functionalization with crosslinking ability, have been well evaluated. In this respect, we have done systematic work to fully illustrate the properties of NLO dendrimers. It is amazing that our $4^{\text {th }}$ and $5^{\text {th }}$ generations of "tree-like" dendrimers displayed an NLO coefficient $\left(d_{33}\right)$ of 177 and $193 \mathrm{pm} \mathrm{V}^{-1}$, respectively ${ }^{21 h}$ just by using a simple azo-benzene chromophore. This record was revised when "global-like" ones achieved higher $d_{33}$ values that peaked at $246 \mathrm{pm} \mathrm{V}^{-1}$ (ref. 22) (Scheme S1 $\dagger$ ). Based on these two types of dendrimer topology, the concept of an "Isolation Chromophore" ${ }^{23}$ and the "Ar-ArF" self-assembly ${ }^{24}$ effect were subsequently utilized to achieve better performance (Schemes S2-S4 $\dagger$ ). Using the same model chromophore but in a different construction form, we have built a database-like system of the structure-property relationships of NLO dendrimers (see the ESI $\dagger$ ). However, we are also confused that their $d_{33}$ values are all around $250 \mathrm{pm} \mathrm{V}^{-1}$, instead of a badly needed new breakthrough. Is this the limit? Or is there still some room for further improvement through the optimization of dendrimer topology? Analyzing all the dendrimers carefully, it was found that the dendrimers in our database, and also other NLO dendrimers reported so far, ${ }^{21}$ are designed monofunctional with good symmetrical patterns (Fig. 1c). The non-centrosymmetric state of the chromophore moieties is realized entirely depending on the poling process. Until now, little research has considered the original molecule structure, no matter whether this might benefit the poling process and maximize the macroscopic second order NLO effect in theory. This thought had initially occurred to us as a clue when we proposed a novel kind of "X"-type compounds possessing high $d_{33}$ values, in which the alignment of the dipole moments of the chromophore moieties is taken into consideration (Scheme S5†). ${ }^{25}$ This undoubtedly represents a promising approach to achieve high NLO efficiency, and prompted us to explore the possibility of whether breaking the symmetry of dendrimers, resulting in Janus dendrimers, could break some new ground. 
In this paper, three Janus dendrimers, $\mathbf{D - 1 3 N}, \mathbf{D - 1 7 N}$ and $\mathbf{D}$ 21N, were prepared (Fig. 1d). Different from many classic Janus dendrimers considered in terms of their different functional end groups, the Janus feature of these three dendrimers here was from the diversity in the dipole $(\mu)$ orientation of two dendrons. That is to say, the final dendrimers have a noncentrosymmetric structure of dipole orientation to some degree. To make this point more clearly, we labelled the two expansion parts as the $x$-axis and anti- $x$-axis dendron. It is easy to distinguish that the dipole orientation of each dendron is in the same direction, the peripheral groups of the $x$-axis dendron are the electron donating part (aniline linked with benzene) and those of the anti- $x$-axis dendron are the electron accepting part (nitrobenzene). Besides, D-13N has 6.5 chromophores in the $x$ axis part (the upper yellow part) and 6.5 chromophores in the anti- $x$-axis part (the lower blue part), D-17N has 14.5 ( $x$-axis) and 2.5 (anti- $x$-axis), while $\mathbf{D}-\mathbf{2 1 N}$ has 14.5 ( $x$-axis) and 6.5 (anti- $x$ axis). It is obvious that $\mathbf{D}-\mathbf{1 3 N}$ has the most balanced structure in terms of generation or chromophore numbers. The diversity of dipole orientation in the two sides endows these dendrimers with a new Janus feature, and the consequent non-centrosymmetric alignment could benefit their second-order NLO performance. Excitingly, all the three dendrimers demonstrate a large NLO coefficient; the $d_{33} @ 1064$ values of D-13N, D-17N and D-21N are 299, 238 and $261 \mathrm{pm} \mathrm{V}^{-1}$, respectively. The $d_{33}$ value of D-21N (261 $\mathrm{pm} \mathrm{V}^{-1}$ ) reaches the first-class level for azobased polymers, and that of $\mathbf{D}-\mathbf{1 3 N}\left(299 \mathrm{pm} \mathrm{V}^{-1}\right)$ is even larger than all of polymers that have ever been reported before, demonstrating the power of the control of molecular topology. This example might shed some light on the design of functional dendrimers with special topology. Herein, we present the synthesis, characterization and properties of these new dendrimers in detail.

\section{Results and discussion}

\section{Synthesis and characterization}

The target dendrimers contain relatively large conjugated molecular fragments of nitro-azobenzene moieties, thus the synthetic method to construct a Janus dendrimer through the reaction of two dendrons was not suitable here. As shown in Fig. 2, we divided D-13N, D-17N and D-21N into small fragments including small molecular fragments and G1-type fragments. First, the anti- $x$-axis dendron was built through the reaction between the fragments and the core by a convergent method. Secondly, the $x$-axis dendron was constructed through a divergent method (Fig. 2a, D-13N; 2b, D-21N; 2c, D$\mathbf{1 7 N}$ ). By the reasonable segmentation of molecular fragments and the utilization of the combination of "converge" and "diverge" methods, the target macromolecules were successfully obtained in satisfactory yields. For example, the click reaction $[\mathrm{Cu}(\mathrm{I})$-catalyzed azide-alkyne cycloaddition (CuAAC) $]^{26}$ utilized in the last step gave high yields: $89.0 \%$ for D-17N, 88.3\% for D-13N, and $87.4 \%$ for D-21N. The detailed synthetic routes are presented in the experimental part in the ESI. $\dagger$ All of these dendrimers could be well dissolved in common polar solvents like $\mathrm{CH}_{2} \mathrm{Cl}_{2}$ and THF, ensuring their

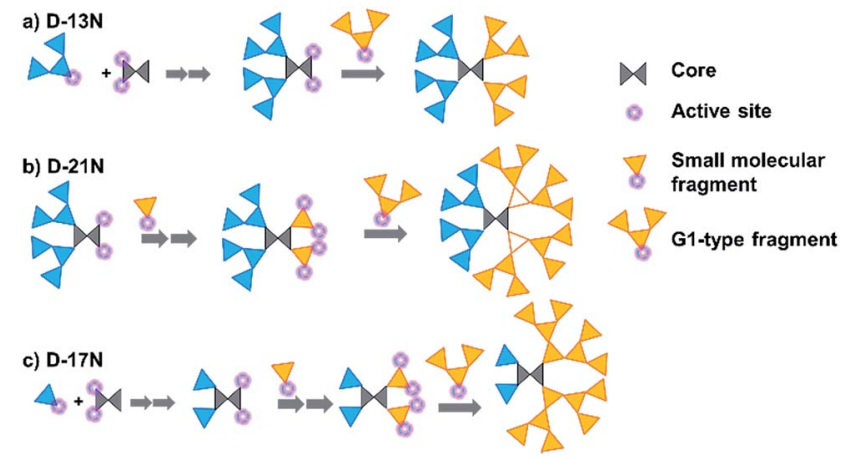

Fig. 2 "Converge-then-diverge" methods to construct Janus dendrimers.

convenient synthesis, purification and processing. In addition, thanks to the high yield and big different polarity of the product from reactants, the target Janus dendrimers were easily purified by simple column chromatography. For example, D-21N had the highest polarity, which meant it could be isolated conveniently by column chromatography using THF as an eluent.

The products were first identified by ${ }^{1} \mathrm{H}-\mathrm{NMR}$ and ${ }^{13} \mathrm{C}-\mathrm{NMR}$ analysis (Fig. S6-S31 $\dagger$ ), which is a powerful and simple method to identify small molecules and low generation dendrons. But for the target dendrimers, the peaks are broad and overlap with each other significantly, making it difficult to confirm exactly their accurate structures from their NMR spectra. MALDI-TOF mass spectrometry and a GPC method were further utilized to characterize the dendrimers, with the results summarized in Table 1 and the ESI. $\uparrow$ The molecular weights of the dendrimers range from 6820 to 11 139, accompanied with an increase in the content of chromophore moieties. All the polydispersity ratios $\left(M_{\mathrm{w}} / M_{\mathrm{n}}\right)$ of these three dendrimers, measured by GPC, were close to 1 , and the peaks were very sharp and narrow, even compared to a polystyrene standard. The trend of $M_{\mathrm{w}}$ values agrees with the real one, but the values were much smaller. ${ }^{27}$ The results could briefly reflect the difference between the $M_{\mathrm{w}}$ value measured by GPC and the real molecular weight, or in other words, the GPC data of dendrimers could be regarded as a reference for the investigation of similar polymeric systems.

All the dendrimers possess good thermal stability as the $T_{\mathrm{d}}$ is located at around $280{ }^{\circ} \mathrm{C}$ (Fig. S36†). The glass transition temperatures $\left(T_{\mathrm{g}}\right)$ of these three dendrimers were also investigated by using differential scanning calorimetry (DSC), however, no obvious peaks were found to determine the exact $T_{\mathrm{g}}$ (Fig. S37 $\dagger$ ). A vibrational spectral (FT-IR) study was conducted (Fig. S38 $\dagger$ ). The vibration peaks of $-\mathrm{N}_{3}$ and $-\mathrm{C}=\mathrm{O}$ were typically detected in the IR spectra. The peak of $-\mathrm{N}_{3}$ appears obviously for the reactant compound while it disappears for the product after the click reaction. The intensities of the $-\mathrm{C}=\mathrm{O}$ peak vary from compound to compound, depending on the content of $-\mathrm{C}=\mathrm{O}$ groups. The peak positions of the nitro-azobenzene chromophore moiety basically remain unchanged before and after the final reaction, partially confirming that the chromophore moieties were stable during the synthetic process. 
Table 1 Characterization data

\begin{tabular}{|c|c|c|c|c|c|c|}
\hline No. & $m / z^{a}$ & $m / z^{b}$ (cal) & $M_{\mathrm{w}}{ }^{c}$ & $M_{\mathrm{w}} / M_{\mathrm{n}}{ }^{c}$ & $T_{\mathrm{d}}^{d}\left({ }^{\circ} \mathrm{C}\right)$ & GPC peaks of dendrimers ${ }^{e}$ \\
\hline D-13N & 6820 & 6827.67 & 6924 & 1.035 & 287 & \\
\hline D-17N & 9145 & 9144.49 & 7745 & 1.052 & 283 & \\
\hline
\end{tabular}

\begin{abstract}
${ }^{a}$ Measured by MALDI-TOF mass spectroscopy. ${ }^{b}$ Calculated for $[\mathrm{M}+\mathrm{Na}]^{+} .{ }^{c}$ Determined by GPC in THF, based on calibration with polystyrene. ${ }^{d}$ The $5 \%$ weight loss temperature of polymers detected by TGA analysis under nitrogen at a heating rate of $10^{\circ} \mathrm{C} \mathrm{min}{ }^{-1}$. ${ }^{e}$ (a) $\mathbf{D}-13 \mathbf{N}$, (b) D-17N, (c) $\mathbf{D}-\mathbf{2 1 N}$ and standard (d) polystyrene (commercially available, $M_{\mathrm{w}}=13880, M_{\mathrm{w}} / M_{\mathrm{n}}=1.02$ ), tested here: $M_{\mathrm{w}}=11940, M_{\mathrm{w}} / M_{\mathrm{n}}=1.08$.
\end{abstract}

\section{Photophysical properties}

The NLO chromophore was designed with a typical $\mathrm{D}-\pi-\mathrm{A}$ structure, whose $\mu \beta$ value is related to the intramolecular charge transfer (ICT) effect. The most convenient and basic method to analyze this is UV-visible spectroscopy. The spectra of the dendrimers and other related compounds are displayed in Fig. S39$\mathrm{S} 43, \uparrow$ with the maximum absorption wavelengths $\left(\lambda_{\max }\right)$ of the target dendrimers listed in Table 2. The main absorptions at $460-480 \mathrm{~nm}$ are attributed to the $\pi \rightarrow \pi^{*}$ transitions of the nitro-azobenzene chromophores, and these three dendrimers share the same chromophore components and display almost the same absorptions.

For comparison, the UV-vis spectrum of $\mathbf{C 1}$, which contains one nitro-azobenzene chromophore moiety (Scheme S7 $\dagger$ ), was tested. Due to the subtle differences between $\mathbf{C} 1$ and the chromophores linked in the dendrimer, $\mathbf{C} \mathbf{1}$ was slightly red-shifted in each kind of solvent. All the compounds displayed solvatochromism phenomena, ${ }^{28}$ that is, the UV-vis spectra and $\lambda_{\max }$ were different in different solvent. But $\mathbf{C 1}$ and the dendrimers exhibit different spectral shifts in each solvent. Setting $\Delta$ as the difference between the $\lambda_{\max }$ values in 1,4-dioxane and DMSO, C1 displays the biggest $\Delta$ value of $34 \mathrm{~nm}$, while all the dendrimers display smaller $\Delta$ values, especially $\mathbf{D}-\mathbf{1 3 N}$ and $\mathbf{D}$ $21 \mathrm{~N}$, with a $\Delta$ value of only $24 \mathrm{~nm}$. This indicates that the impact of solvent on chromophore moieties in dendrimers is much different from that in small molecules, or in other words, the separated small chromophore molecules were much more easily affected by solvent than the chromophore moieties

Table 2 The maximum absorption wavelength $\left(\lambda_{\max }, \mathrm{nm}\right)$ in different solvents $\left(0.02 \mathrm{mg} \mathrm{mL}^{-1}\right)^{a}$

\begin{tabular}{llllllll}
\hline & 1,4-dioxane & $\mathrm{CHCl}_{3}$ & THF & $\mathrm{CH}_{2} \mathrm{Cl}_{2}$ & DMF & DMSO & $\Delta$ \\
\hline D-13N & 458 & 458 & 463 & 461 & 476 & 482 & 24 \\
D-17N & 455 & 458 & 460 & 459 & 473 & 482 & 27 \\
D-21N & 457 & 457 & 462 & 458 & 474 & 481 & 24 \\
C1 & 464 & 474 & 470 & 476 & 488 & 498 & 34
\end{tabular}

${ }^{a} \Delta=\lambda_{\max }$ (in DMSO) $-\lambda_{\max }$ (in 1,4-dioxane). hidden in dendrimers. This highly reduced solvatochromic shift is called the "site-isolation effect", which typically exists in polymers containing bulk isolation groups, ${ }^{29}$ due to the shielding effect of the bulky groups on the chromophore moieties in polymers. Here for these dendrimers, there were no bulky groups but all of them demonstrated an obvious "siteisolation effect", which was supposed to be derived from their unique branched structure.

\section{NLO properties}

The NLO effect originates from the non-centrosymmetric alignment of the chromophore moieties, which could be conveniently achieved by using a corona-poling method to fabricate the poled thin films. The NLO effect could be quantified as a $d_{33}$ value by testing the double-frequency signals generated from the poled films when using a second harmonic generation (SHG) technique (the calculation method is presented in the ESI $\dagger$ ). In the poling process, the film was heated gradually under a highvoltage electric field at a temperature approaching the best poling temperature of $T_{\mathrm{e}}$ (approximately the glass transition temperature). At $T_{\mathrm{e}}$, there is an equilibrium state between the random motion of molecules and the ordered arrangement driven by the electric field, and this could be characterized by the output double-frequency signal in real time.

The dendrimers were spin-coated onto glass substrates to form $200-250 \mathrm{~nm}$ thick films to test their NLO performance (details of NLO measurements are presented in the ESI $\dagger$ ), with the characterization parameters listed in Table 3. The basic measurement was carried out by using a fundamental beam of $1064 \mathrm{~nm}$ and a double-frequency beam of $532 \mathrm{~nm}$ was detected. Through taking measurements many times, it was confirmed that these three dendrimers demonstrate very large NLO coefficients. The $d_{33} @ 1064$ values of D-13N, D-17N and D-21N are 299, 238 and $261 \mathrm{pm} \mathrm{V}^{-1}$, respectively, where the $d_{33}$ value of $\mathbf{D}$ $21 \mathrm{~N}\left(261 \mathrm{pm} \mathrm{V}^{-1}\right)$ reaches the first class level for azo-based polymers, and that of $\mathbf{D}-\mathbf{1 3 N}\left(299 \mathrm{pm} \mathrm{V}^{-1}\right)$ is even larger than all of polymers containing azo chromophores that have ever been reported. D-17N demonstrates the smallest $d_{33}$ value in this system, but this is still very large $\left(238 \mathrm{pm} \mathrm{V}^{-1}\right)$. 
Table 3 The NLO properties of dendrimers

\begin{tabular}{|c|c|c|c|c|c|c|c|c|}
\hline & $T_{\mathrm{e}}^{a}\left({ }^{\circ} \mathrm{C}\right)$ & $\lambda_{\max }^{b}(\mathrm{~nm})$ & $d_{33}{ }^{c} @ 1064\left(\mathrm{pm} \mathrm{V}^{-1}\right)$ & $d_{33(\infty)}{ }^{d} @ 1064\left(\mathrm{pm} \mathrm{V}^{-1}\right)$ & $d_{33}{ }^{c} @ 1950\left(\mathrm{pm} \mathrm{V}^{-1}\right)$ & $d_{33(\infty)}{ }^{d} @ 1950\left(\mathrm{pm} \mathrm{V}^{-1}\right)$ & $\Phi^{e}$ & $N^{f}(\%)$ \\
\hline D-13N & 105 & 476 & $299(28)$ & 48 & $73(8)$ & 52 & 0.24 & 56.9 \\
\hline D-17N & 110 & 471 & $238(20)$ & 41 & $63(7)$ & 45 & 0.19 & 55.5 \\
\hline
\end{tabular}

${ }^{a}$ The best poling temperature. ${ }^{b}$ The maximum absorption in thin films. ${ }^{c}$ Second harmonic generation (SHG) coefficient with the standard deviation in brackets. ${ }^{d}$ The nonresonant $d_{33}$ values calculated by using the approximate two-level model. ${ }^{e}$ Order parameter $\Phi=1-A_{1} / A_{0}, A_{1}$ and $A_{0}$ are the absorbance of the polymer film after and before corona poling, respectively. ${ }^{f}$ The loading density of the effective chromophores.

In addition, we calculated the average deviations and standard deviations based on many test results (see error analysis in the ESI $\dagger$ ). The standard deviations of $d_{33} @ 1064$ are 28, 20 and $22 \mathrm{pm} \mathrm{V}^{-1}$ for $\mathbf{D}-\mathbf{1 3 N}, \mathbf{D}-\mathbf{1 7 N}$ and $\mathbf{D}-\mathbf{2 1 N}$, with relative standard deviations of $9.5 \%, 8.3 \%$, and $8.4 \%$ respectively. The relative average deviations and relative standard deviations are all below $10 \%$.

To further evaluate the NLO efficiency of these dendrimers, SHG tests were also conducted under another fundamental beam of $1950 \mathrm{~nm}$. The $d_{33} @ 1950$ values were 73, 63 and $69 \mathrm{pm}$ $\mathbf{V}^{-1}$ for $\mathbf{D - 1 3 N}, \mathbf{D - 1 7 N}$ and $\mathbf{D - 2 1 N}$, respectively. The standard deviations of $d_{33}$ @1950 are 8, 7 and $10 \mathrm{pm} \mathrm{V}^{-1}$ for $\mathbf{D}-\mathbf{1 3 N}, \mathbf{D}$ $17 \mathbf{N}$ and $\mathbf{D - 2 1 N}$, with relative standard deviations of $11.0 \%$, $12.2 \%$, and $14.6 \%$, respectively. The $d_{33}$ @1950 values are much smaller than the $d_{33} @ 1064$ values due to the dispersion of the $d_{33}$ values, ${ }^{18 a}$ but they agree with the corresponding $d_{33} @ 1064$ values. For our recent systems measured under a $1950 \mathrm{~nm}$ laser, the value around $70 \mathrm{pm} \mathrm{V}^{-1}$ was a very large one, indicating that $\mathbf{D - 1 3 N}$ and $\mathbf{D - 2 1 N}$ are really good NLO materials with large NLO coefficients. To further confirm these results, some compounds in our previous work were measured at the same time; the results of D-13N, D-17N and D-21N stand out from them (see error analysis in the ESI $\dagger$ ). In addition, the $d_{33(\infty)}$ value was calculated by an approximate two-level model (eqn (S9) $\dagger$ ), which subtracted out the impact of the fundamental beam..$^{30}$ As a result, the value of $d_{33(\infty)}$ 1064 was close to that of $d_{33(\infty)}$ 1950 for each dendrimer.

According to the results of the SHG measurements under the fundamental beams of both 1064 and $1950 \mathrm{~nm}$, it was exciting that all the Janus dendrimers demonstrate very high NLO coefficients. It is more interesting that $\mathbf{D - 1 3 N}$ and $\mathbf{D - 2 1 N}$ display significantly larger coefficients than $\mathbf{D - 1 7 N}$. The three dendrimers are all Janus type, which is supposed to be the intrinsic reason for achieving high NLO coefficients, but they have Janus features to different extents, as demonstrated in the unbalanced number of dendritic generations in the two sides.

Except for the difference in $d_{33}$ values of each dendrimer and the solvatochromism phenomena discussed above, there are other differences from the poling process observed. The order parameter $(\Phi)$ is a semiquantitative factor to judge the poling extent of thin films. ${ }^{31}$ The $\Phi$ values of D-13 (0.24) and D21N (0.22) are obviously larger than that of $\mathbf{D - 1 7 N}(0.19)$, indicating that the thin films of $\mathbf{D - 1 3 N}$ and $\mathbf{D - 2 1 N}$ were better poled than that of $\mathbf{D - 1 7 N}$. This could be also shown by their poling and de-poling curves (Fig. 3a-c, method is presented in the ESI $\dagger$ ). A poling curve came from the signals when the poled film was heated gradually (at a heating rate of $3{ }^{\circ} \mathrm{C} \mathrm{min}^{-1}$ ). D-17N and D-21N have a narrow band of poling temperature ( $T_{\mathrm{e}}$ band), during which the films could be greatly poled. However, D-13N shows a very wide $T_{\mathrm{e}}$ band from $100{ }^{\circ} \mathrm{C}$ to $130{ }^{\circ} \mathrm{C}$. To some extent, $\mathbf{D - 1 3 N}$ was relatively easier to be poled. On the other hand, the de-poling curve came from the signals when the poled film was heated $\left(3{ }^{\circ} \mathrm{C} \mathrm{min}^{-1}\right)$ without an electric field. The NLO thermal stability could be evaluated according to the de-poling curve. The signal decayed at about $90{ }^{\circ} \mathrm{C}$ for $\mathbf{D}-\mathbf{1 7 N}, 100{ }^{\circ} \mathrm{C}$ for $\mathbf{D}-\mathbf{1 3 N}$, and $113{ }^{\circ} \mathrm{C}$ for $\mathbf{D}-21 \mathbf{N}$. The trend of $T_{\mathrm{dp}}$ roughly agrees with the trend of $T_{\mathrm{e}}$. For most polymers or dendrimers, $T_{\mathrm{dp}}$ is lower than $T_{\mathrm{e}}{ }^{16-18} \mathrm{Just}$ as in the case of $\mathbf{D}-\mathbf{1 7 N}$, the $T_{\mathrm{dp}}$ band has no intersection with the $T_{\mathrm{e}}$ band. But interestingly, for $\mathbf{D}-\mathbf{1 3 N}$ and $\mathbf{D}-\mathbf{2 1 N}$, the $T_{\mathrm{dp}}$ band overlaps with the $T_{\mathrm{e}}$ band, especially for D-21N. That is to say, D-13N and D-21N have a better capability to maintain the alignment of dipole moments, which conforms to the original intention of our design.

To find out their spatial conformation, we used a molecular mechanics simulation method to calculate these dendrimers, which is widely used in many cases for polymers ${ }^{32}$ and in our previous study. ${ }^{25}$ The molecular weights of the dendrimers range from 6800 to 11000 , for which the molecular mechanics simulation is suitable and effective. Calculation details and images viewed from different angles as presented in the ESI $\dagger$ and Fig. 3d-f give a general structure of these three Janus dendrimers. The structure-property relationships of the Janus system could be discussed in two aspects.

Firstly, all the three dendrimers share a same "oriented" Janus system, whose feature is the diversity in dipole orientation of the dendrons in the two sides, which contribute to the good NLO performance. As seen in the structures of the three dendrimers, the yellow part and the blue part of the dendrons are non-centrosymmetric (Fig. 1d). Because of their special natural structure with the chromophores arranged in a particular order, they exhibit a large NLO efficiency. The specific dipole orientation of two dendrons gives a non-centrosymmetric alignment of the dipole moment in the whole dendrimers with the aid of the poling process, and the noncentrosymmetry ensures good macroscopic NLO performance. The results confirm the success and validity of the introduction of a Janus system into the molecular design of NLO dendrimers.

Secondly, D-17N and D-21N have a "generational" Janus system, whose feature is the diversity in the number of dendritic 


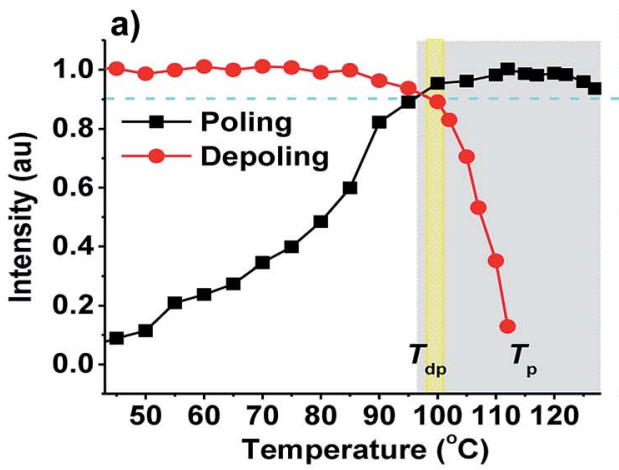

d)

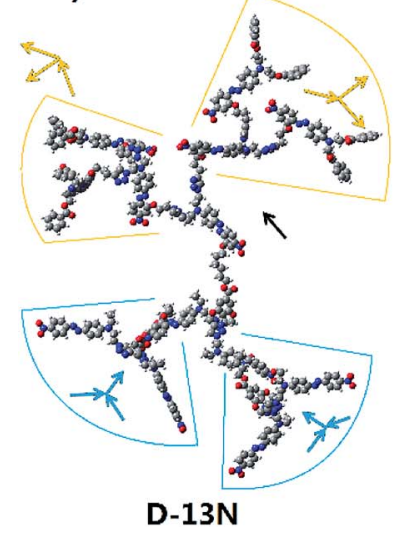

b)

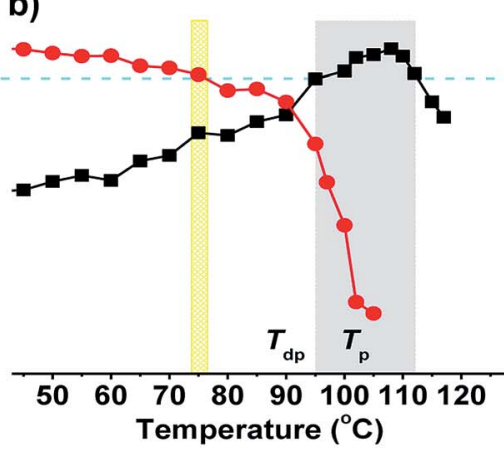

c)

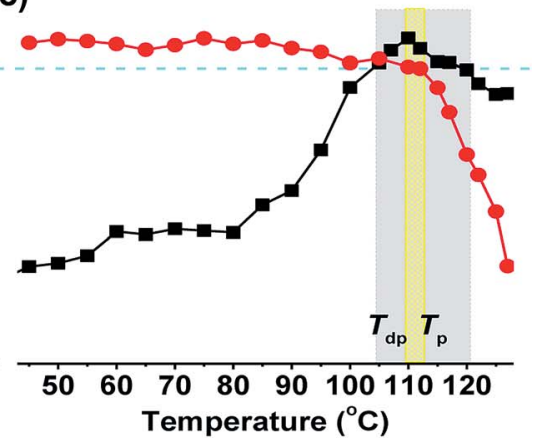

e)

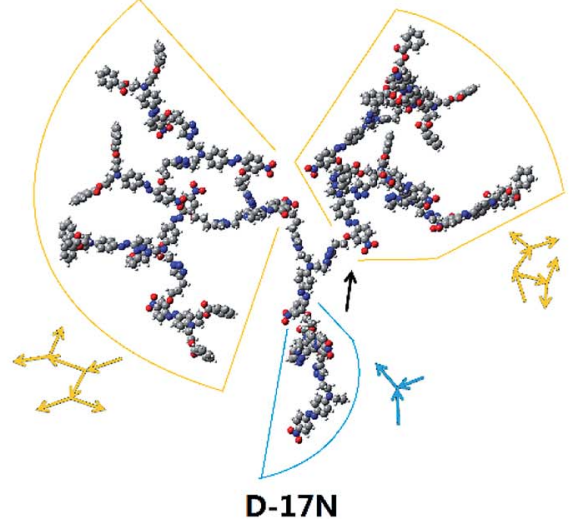

f)

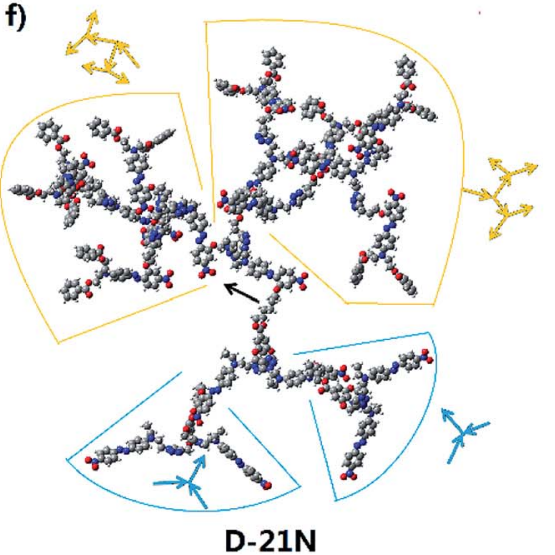

Fig. 3 Poling and de-poling curves of (a) D-13N, (b) D-17N and (c) D-21N and calculated structures of (d) D-13N, (e) D-17N and (f) D-21N.

generations in the two sides. In this respect, $\mathbf{D - 1 7} \mathbf{N}$ is the most unbalanced dendrimer with a "generational" Janus feature, but D-13N does not have this diversity. As shown in Fig. 3d and e, D-13N and D-21N have an "X"-type structure, especially $\mathbf{D - 1 3 N}$, while in Fig. 3 f shows that $\mathbf{D - 1 7 N}$ is more like a "Y". As designed, D-13N or D-21N has a large anti- $x$-axis dendron part, which leaves the $x$-axis dendron with not enough room to offset the dipole moment caused by the anti- $x$-axis part and the possibility of keeping the orientation against the anti- $x$-direction. Thus the alignment of the chromophores can be maintained. But for $\mathbf{D}$ $\mathbf{1 7 N}$, its $x$-axis dendron is large, while the anti- $x$-axis dendron is small, which gives the chains of the $x$-axis dendron enough room to draw toward the anti- $x$-axis direction in order to reduce the total dipole moment. The calculated conformation could help us to confirm the disadvantage of a "generational" Janus system in the NLO field. There is also some difference between D-13N and D-21N; D-21N has a larger $x$-axis dendron part, meanwhile D-13N seems more flexible. This might be the key reason that D-13N was more easily poled (Fig. 3a). As for D-21N, this was hard to be poled, and accordingly, it was hard to decay once poled (Fig. 3c).

Both the advantages of the "oriented" Janus system and the disadvantages of the "generational" Janus system make it clear that the key point to enhance the NLO efficiency of dendrimers, in terms of the controllable topology, is the noncentrosymmetric alignment of dipole orientation. Therefore, a new molecular design idea, which combines our previous work focusing on the centrosymmetric growth of dendritic generation and the non-centrosymmetric dipole orientation of various dendrons discussed in this paper, has become a promising direction in the field of NLO materials.

Actually, in comparison with the dendrimers in the previous study (Schemes S1-S4†), D-13N, D-17N and D-21N are not really high-generation dendrimers, and their synthesis was relatively much easier. Considering the NLO coefficient of the dendrimers and their generation (or numbers of chromophore moieties that they contain), we roughly divided these values into four quadrants (I, II, III and IV in Fig. 4). Most NLO dendrimers are located in quadrant III, where low generation corresponds to $d_{33}$ values lower than $240 \mathrm{pm} \mathrm{V}^{-1}$, while a few are located in quadrant I, where dendrimers exhibit high NLO coefficients and have high-generation obtained through heavy synthesis. Dendrimers in quadrant IV are obtained through a tough synthesis but possess an imbalanced NLO efficiency. Quadrant II is the region where dendrimers exhibit high NLO coefficients with low-generation. However, there were no dendrimers located in quadrant II before D-13N and D-21N were obtained here. The synthesis of D-13N and D-21N was easier when compared to that of the dendrimers in quadrant I. Since the synthetic process has been investigated and improved in this paper, it will be even more convenient to obtain dendrimers in this form. In addition, it might provide certain enlightenment and a reference for us in establishing a new system that combines a Janus topology structure with beneficial factors (like Ar-ArF self-assembly and isolation chromophore effect) in future research. 


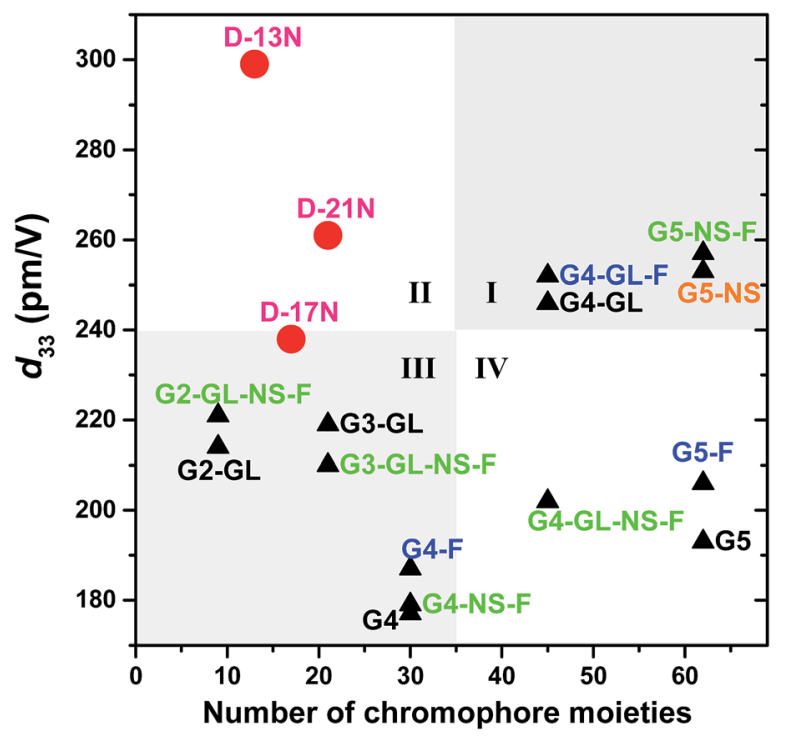

Fig. 4 Four quadrants: $d_{33}$ value related to amount of chromophore moieties.

\section{Conclusions}

In summary, we have demonstrated the possibility of further improving NLO efficiency by constructing a new type of Janus dendrimers. The dendrimers contain $x$-axis and anti-x-axis dendrons, where the alignment of the chromophore moieties is taken into consideration. The target dendrimers D-13N, D-17N and D-21N demonstrate extremely large NLO coefficients of 299, 238 and $261 \mathrm{pm} \mathrm{V}^{-1}$, respectively. The perfect Janus structure is supposed to be the main reason for achieving the large NLO efficiency. The $d_{33}$ value (up to $299 \mathrm{pm} \mathrm{V}^{-1}$ ) of $\mathbf{D}-\mathbf{1 3 N}$ is the highest value reported so far for polymers containing a simple azo-chromophore moiety. Thus, these successful examples open up a new avenue in constructing Janus dendrimers with good NLO properties, and perhaps shed some light on other functional dendrimers with special requirements for their molecular topology.

\section{Acknowledgements}

We are grateful to the National Science Foundation of China (no. 21325416) for financial support.

\section{References}

1 (a) H. Ringsdorf, B. Schlarb and J. Venzmer, Angew. Chem., Int. Ed. Engl., 1988, 27, 113-158; (b) J. M. J. Fréchet, Science, 1994, 263, 1710-1715; (c) N. Hadjichristidis, M. Pitsikalis, S. Pispas and H. Iatrou, Chem. Rev., 2001, 101, 3747-3792; (d) B. A. Hammer and K. Mullen, Chem. Rev., 2016, 116, 2103-2140; (e) W. Wu, R. Tang, Q. Li and Z. Li, Chem. Soc. Rev., 2015, 44, 3997-4022.

2 (a) D. Aoki, S. Uchida and T. Takata, Angew. Chem., 2015, 127, 6874-6878; Angew. Chem., Int. Ed., 2015, 54, 6770; (b) R. Wang, W. Yuan and X. Zhu, Chin. J. Polym. Sci., 2015,
33, 680-687; (c) R. Qin, Y. Jiang, H. Zhang, K. Zhang, Q. Zhang and F. Chang, Chin. J. Polym. Sci., 2015, 33, 490498; (d) Z. Zhang and Y. Li, Sci. China: Chem., 2015, 58, 192-209; (e) G. Pasparakis, N. Krasnogor, L. Cronin, B. G. Davis and C. Alexander, Chem. Soc. Rev., 2010, 39, 286-300.

3 (a) C. C. Lee, J. A. MacKay, J. M. Frechet and F. C. Szoka, Nat. Biotechnol., 2005, 23, 1517-1526; (b) D. Astruc, Nat. Chem., 2012, 4, 255-267; (c) A. M. Caminade, D. Yan and D. K. Smith, Chem. Soc. Rev., 2015, 44, 3870-3873; (d) Z. Qiao and X. Shi, Prog. Polym. Sci., 2015, 44, 1-27.

4 (a) C. J. Hawker and J. M. J. Frechet, Macromolecules, 1990, 23, 4726-4729; (b) I. J. Majoros, A. Myc, T. Thomas, C. B. Mehta and J. R. Baker Jr, Biomacromolecules, 2006, 7, 572-579; (c) B. A. Hammer, R. Moritz, R. Stangenberg, M. Baumgarten and K. Mullen, Chem. Soc. Rev., 2015, 44, 4072-4090.

5 (a) C. C. Lee, E. R. Gillies, M. E. Fox, S. J. Guillaudeu, J. M. Frechet, E. E. Dy and F. C. Szoka, Proc. Natl. Acad. Sci. U. S. A., 2006, 103, 16649-16654; (b) A.-M. Caminade, R. Laurent, B. Delavaux-Nicot and J.-P. Majoral, New J. Chem., 2012, 36, 217-226.

6 A. Sharma, K. Neibert, R. Sharma, R. Hourani, D. Maysinger and A. Kakkar, Macromolecules, 2011, 44, 521-529.

7 T. Qin, W. Wiedemair, S. Nau, R. Trattnig, S. Sax, S. Winkler, A. Vollmer, N. Koch, M. Baumgarten, E. J. List and K. Mullen, J. Am. Chem. Soc., 2011, 133, 1301-1303.

8 R. Sharma, N. Kottari, Y. M. Chabre, L. Abbassi, T. C. Shiao and R. Roy, Chem. Commun., 2014, 50, 13300-13303.

9 (a) E. R. Gillies and J. M. J. Fréchet, J. Am. Chem. Soc., 2002, 124, 14137-14146; (b) X. Feng, D. Taton, E. Ibarboure, E. L. Chaikof and Y. Gnanou, J. Am. Chem. Soc., 2008, 130, 11662-11676.

10 (a) V. Percec, M. R. Imam, M. Peterca and P. Leowanawat, J. Am. Chem. Soc., 2012, 134, 4408-4420; (b) J. Mikkilä, H. Rosilo, S. Nummelin, J. Seitsonen, J. Ruokolainen and M. A. Kostiainen, ACS Macro Lett., 2013, 2, 720-724.

11 (a) I. J. Majoros, A. Myc, T. Thomas, C. B. Mehta and J. R. Baker Jr, Biomacromolecules, 2006, 7, 572-579; (b) L. Sun, X. Ma, C. M. Dong, B. Zhu and X. Zhu, Biomacromolecules, 2012, 13, 3581-3591.

12 (a) C. Ornelas, R. Pennell, L. F. Liebes and M. Weck, Org. Lett., 2008, 10, 4751-4754; (b) C. Dengiz, B. Breiten, J. P. Gisselbrecht, C. Boudon, N. Trapp, W. B. Schweizer and F. Diederich, J. Org. Chem., 2015, 80, 882-896.

13 (a) M. C. Oh, H. Zhang, A. Szep, V. Chuyanov, W. H. Steier, C. Zhang, L. R. Dalton, H. Erlig, B. Tsap and H. R. Fetterman, Appl. Phys. Lett., 2000, 76, 3525-3528; (b) C. Haffner, W. Heni, Y. Fedoryshyn, J. Niegemann, A. Melikyan, D. L. Elder, B. Baeuerle, Y. Salamin, A. Josten, U. Koch, C. Hoessbacher, F. Ducry, L. Juchli, A. Emboras, D. Hillerkuss, M. Kohl, L. R. Dalton, C. Hafner and J. Leuthold, Nat. Photonics, 2015, 9, 525-528.

14 S. I. Bozhevolnyi, V. S. Volkov, E. Devaux, J. Y. Laluet and T. W. Ebbesen, Nature, 2006, 440, 508-511.

15 C. Sun, Z. Li, C. Zheng, Q. Luo, X. Huang and D. Zhang, Opt. Commun., 2014, 331, 251-261. 
16 (a) S. K. Yesodha, C. K. Sadashiva Pillai and N. Tsutsumi, Prog. Polym. Sci., 2004, 29, 45-74; (b) L. R. Dalton, P. A. Sullivan and D. H. Bale, Chem. Rev., 2010, 110, 25-55; (c) J. Luo, S. Huang, Z. Shi, B. M. Polishak, X. Zhou and A. K. Y. Jen, Chem. Mater., 2011, 23, 544-553.

17 (a) S. R. Marder, B. Kippelen, A. K. Y. Jen and N. Peyghambarian, Nature, 1997, 388, 845-851; (b) Y. Enami, C. T. Derose, D. Mathine, C. Loychik, C. Greenlee, R. A. Norwood, T. D. Kim, J. Luo, Y. Tian, A. K. Y. Jen and N. Peyghambarian, Nat. Photonics, 2007, 1, 180-185; (c) C. Ji, Z. Sun, S. Zhang, S. Zhao, T. Chen, Y. Tang and J. Luo, Chem. Commun., 2015, 51, 2298-2300.

18 (a) K. D. Singer, M. G. Kuzyk and J. E. Sohn, J. Opt. Soc. Am. B, 1987, 4, 968-976; (b) S. M. Risser, D. N. Beratan and S. R. Marder, J. Am. Chem. Soc., 1993, 115, 7719-7728.

19 (a) G. R. Meredith, J. VanDusen and D. J. Williams, Macromolecules, 1982, 15, 1385-1389; (b) M. A. Hubbard, T. J. Marks, J. Yang and G. K. Wong, Chem. Mater., 1989, 1, 167-169; (c) M. Ahlheim, M. Barzoukas, P. V. Bedworth, M. Blanchard-Desce, A. Fort, Z. Y. Hu, S. R. Marder, J. W. Perry, C. Runser, M. Staehelin and B. Zysset, Science, 1996, 271, 335-337.

20 (a) A. Facchetti, E. Annoni, L. Beverina, M. Morone, P. Zhu, T. J. Marks and G. A. Pagani, Nat. Mater., 2004, 3, 910-917; (b) W. Wu, J. Qin and Z. Li, Polymer, 2013, 54, 4351-4382; (c) W. Wu, C. Wang, Q. Li, C. Ye, J. Qin and Z. Li, Sci. Rep., 2014, 4, 6101; (d) W. Jin, P. V. Johnston, D. L. Elder, K. T. Manner, K. E. Garrett, W. Kaminsky, R. Xu, B. H. Robinson and L. R. Dalton, J. Mater. Chem. C, 2016, 4, 3119-3124.

21 (a) S. Yokoyama, T. Nakahama, A. Otomo and S. Mashiko, J. Am. Chem. Soc., 2000, 122, 3174-3181; (b) H. Ma and A. K. Y. Jen, Adv. Mater., 2001, 13, 1201-1205; (c) H. Ma, S. Liu, J. Luo, S. Suresh, L. Liu, S. H. Kang, M. Haller, T. Sassa, L. R. Dalton and A. K. Y. Jen, Adv. Funct. Mater., 2002, 12, 565-574; (d) P. A. Sullivan, H. Rommel, Y. Liao, B. C. Olbricht, A. J. Akelaitis, K. A. Firestone, J. W. Kang, J. Luo, J. A. Davies, D. H. Choi, B. E. Eichinger, P. J. Reid, A. Chen, A. K. Jen, B. H. Robinson and L. R. Dalton, J. Am.
Chem. Soc., 2007, 129, 7523-7530; (e) M. J. Cho, D. H. Choi, P. A. Sullivan, A. J. P. Akelaitis and L. R. Dalton, Prog. Polym. Sci., 2008, 33, 1013-1058; (f) X.-H. Zhou, J. Luo, S. Huang, T.-D. Kim, Z. Shi, Y.-J. Cheng, S.-H. Jang, D. B. Knorr, R. M. Overney and A. K. Y. Jen, Adv. Mater., 2009, 21, 1976-1981; (g) Z. Li, W. Wu, Q. Li, G. Yu, L. Xiao, Y. Liu, C. Ye, J. Qin and Z. Li, Angew. Chem., Int. Ed., 2010, 49, 2763-2767; (h) W. Lin, Y. Cui, J. Gao, J. Yu, T. Liang and G. Qian, J. Mater. Chem., 2012, 22, 9202-9208.

22 W. Wu, L. Huang, C. Song, G. Yu, C. Ye, Y. Liu, J. Qin, Q. Li and Z. Li, Chem. Sci., 2012, 3, 1256-1261.

23 W. Wu, C. Li, G. Yu, Y. Liu, C. Ye, J. Qin and Z. Li, Chem.-Eur. J., 2012, 18, 11019-11028.

24 (a) W. Wu, G. Yu, Y. Liu, C. Ye, J. Qin and Z. Li, Chem.-Eur. J., 2013, 19, 630-641; (b) W. Wu, C. Ye, J. Qin and Z. Li, ACS Appl. Mater. Interfaces, 2013, 5, 7033-7041.

25 R. Tang, S. Zhou, W. Xiang, Y. Xie, H. Chen, Q. Peng, G. Yu, B. Liu, H. Zeng, Q. Li and Z. Li, J. Mater. Chem. C, 2015, 3, 4545-4552.

26 (a) H. C. Kolb, M. G. Finn and K. B. Sharpless, Angew. Chem., Int. Ed., 2001, 40, 2004-2021; (b) V. V. Rostovtsev, L. G. Green, V. V. Fokin and K. B. Sharpless, Angew. Chem., 2002, 114, 2708-2711; Angew. Chem., Int. Ed., 2002, 41, 2596; (c) D. Fournier, R. Hoogenboom and U. S. Schubert, Chem. Soc. Rev., 2007, 36, 1369-1380.

27 M. W. Weimer, J. M. J. Fréchet and I. Gitsov, J. Polym. Sci., Part A: Polym. Chem., 1998, 36, 955-970.

28 C. Reichardt, Chem. Soc. Rev., 1992, 21, 147-153.

29 Q. Li, Z. Li, C. Ye and J. Qin, J. Phys. Chem. B, 2008, 112, 49284933.

30 M. Kauranen, C. Boutton, T. Verbiest, M. N. Teerenstra, K. Clays, A. J. Schouten, R. J. M. Nolte and A. Persoons, Science, 1995, 270, 966-969.

31 J. Luo, S. Liu, M. Haller, L. Liu, H. Ma and A. K. Y. Jen, Adv. Mater., 2002, 14, 1763-1768.

32 (a) W. D. Tian and Y. Q. Ma, Chem. Soc. Rev., 2013, 42, 705727; (b) P. M. Paulo, J. N. Lopes and S. M. Costa, J. Phys. Chem. B, 2007, 111, 10651-10664; (c) G. Pandav and V. Ganesan, J. Phys. Chem. B, 2014, 118, 10297-10310. 